\title{
PERFIL FUNCIONAL DE ATLETAS DE BASQUETEBOL COM TRAUMATISMO DA MEDULA ESPINAL DE ACORDO COM A CIF
}

FUNCTIONAL PROFILE OF WHEELCHAIR BASKETBALL ATHLETES WITH SPINAL CORD

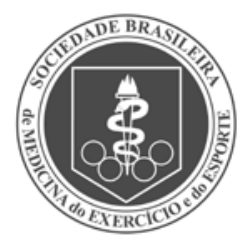

Artigo Original INJURY ACCORDING TO THE ICF

\author{
PERFIL FUNCIONAL DE ATLETAS DE BÁSQUETBOL CON TRAUMATISMO DE LA \\ MÉDULA ESPINAL DE ACUERDO CON LA CIF
}

\author{
Andersom Ricardo Fréz \\ (Fisioterapeuta) \\ Andrezza Thimoteo de Souza \\ (Fisioterapeuta) $^{1}$ \\ Cintia Raquel Bim Quartiero \\ (Fisioterapeuta) ${ }^{1}$ \\ João Afonso Ruaro

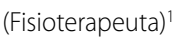

\section{Departamento de Fisioterapia da Universidade Estadual do Centro-Oeste (UNICENTRO), Guarapuava, PR, Brasil.}

\section{Correspondência:}

Av. Simeão Camargo Varela de Sá, 03.85040-080. Guarapuava, PR, Brasil. andersom_frez@yahoo.com.br

\section{RESUMO}

Introdução: A Classificação Internacional de Funcionalidade, Incapacidade e Saúde considera os múltiplos aspectos da funcionalidade; acredita-se que essa ferramenta possa contribuir para avaliar atletas com incapacidades. Objetivo: Determinar o perfil funcional de jogadores de basquete em cadeira de rodas de acordo com o core set da Classificação Internacional de Funcionalidade, Incapacidade e Saúde para indivíduos com traumatismo da medula espinal (TME). Métodos: Foi realizado um estudo transversal com 12 atletas com TME, jogadores de basquete em cadeira de rodas. A funcionalidade foi classificada pela versão abreviada do core set para indivíduos com TME crônico, que contém 33 categorias. Estas foram consideradas válidas se apresentassem ao menos 20\% dos atletas com algum grau de incapacidade (de acordo com os qualificadores da CIF). Resultados: Das 33 categorias avaliadas, 31 (93,9\%) foram representativas para a amostra. Apenas as categorias b710 e e340 não foram consideradas válidas. Em sete categorias todos os atletas apresentaram algum grau de comprometimento (s120, d455, d465, d530, e115, e150 e e580). Porém, os maiores comprometimentos foram observados nas categorias: s120,d455, d465, e115 e e120. Conclusão: Os atletas apresentaram diferentes comprometimentos funcionais, entretanto tais limitações não os impedem de praticar a modalidade esportiva.

Palavras-chave: traumatismos da medula espinal; classificação internacional de funcionalidade, incapacidade e saúde, avaliação em saúde.

\begin{abstract}
Background: The International Classification of Functioning, Disability and Health considers multiples aspects offunctionality; it is believed that this tool can help to evaluate the athletes with disabilities. Objective: To determinate the functional profile of basketball players in wheelchairs according to the International Classification of Functioning, Disability and Health for individuals with spinal cord injury (SCI). Methods: A cross-sectional study was conducted with 12 wheelchair basketball athletes. The functionality has been classified by the abbreviated version of the core sets for individuals with chronic SCl, which contains 33 categories. These categories were considered valid if they had at least 20\% of the athletes with some degree of disability (according to the ICF qualifiers). Results: Of the 33 evaluated categories, 31 (93.9\%) were representative for the sample. Two categories were not considered valid (b710 and e340). In seven categories all athletes had some impairment (s120,d455,d465, d530, e115, e150 e e580). However, the largest impairment was observed in the categories: s120,d455, d465, e115 and e120. Conclusion: The athletes had different functional impairments; however such limitations do not prevent them from practicing the sport.
\end{abstract}

Keywords: spinal cord injuries; international classification of functioning, disability and health, health evaluation.

\section{RESUMEN}

Introducción: La Clasificación Internacional de Funcionalidad, Incapacidad y Salud considera los múltiples aspectos de la funcionalidad; se cree que esa herramienta pueda contribuir para evaluar a atletas con incapacidades. Objetivo: Determinar el perfil funcional de jugadores de básquet en silla de ruedas de acuerdo con el core set de la Clasificación Internacional de Funcionalidad, Incapacidady Salud para individuos con traumatismo de la médula espinal (TME). Métodos: Fue realizado un estudio transversal con 12 atletas con TME, jugadores de básquet en silla de ruedas. La funcionalidad fue clasificada por la versión abreviada del core set para individuos con TME crónico, que contiene 33 categorías. Estas fueron consideradas válidas si presentasen al menos 20\% de los atletas con algún grado de incapacidad (de acuerdo con los calificadores de la (IF). Resultados: De las 33 categorías evaluadas, 31 (93,9\%) fueron representativas para la muestra. Sólo las categorías b710 y e340 no fueron consideradas válidas. En siete categorías todos los atletas presentaron algún grado de limitación (s120, d455, d465, d530, e115, e150 y e580). No obstante, las mayores limitaciones fueron observadas en las categorías: s120, d455, d465, el15 y e120. Conclusión: Los atletas presentaron diferentes limitaciones funcionales, pero tales limitaciones no les impiden practicar la modalidad deportiva.

Palabras clave: traumatismos de la médula espinal, clasificación internacional de funcionalidad, incapacidad y salud, evaluación en salud. 


\section{INTRODUÇÃO}

O basquete em cadeira de rodas (BCR) é um dos mais populares esportes coletivos para usuários de cadeira de rodas ${ }^{1,2}$. Segundo a Federação Internacional de Basquete em Cadeira de Rodas (IWBF) existem mais de 30.000 jogadores no mundo', e dentre estes, estão os indivíduos com traumatismos da medula espinal (TME) ${ }^{2}$.

O TME é uma condição de saúde crônica na qual o indivíduo perde determinadas funções, já que não é capaz de exercê-las da mesma forma como antes da lesão ${ }^{3}$. Entretanto, estas disfunções não determinam completamente a experiência vivida com a incapacidade após o TME, pois isso depende de fatores contextuais, como os fatores ambientais e pessoais ${ }^{4,5}$. Para compreender a experiência vivenciada pelas pessoas com TME é necessária uma abordagem que vai além dos parâmetros biomédicos, e deve englobar as atividades e participação, além dos fatores contextuais ${ }^{3,6}$.

Desde 2001, com a aprovação Classificação Internacional de Funcionalidade, Incapacidade e Saúde (CIF) pela Organização Mundial da Saúde (OMS), o entendimento da funcionalidade e da incapacidade humana passaram a uma nova dimensão ao identificar não apenas a funcionalidade, mas também o ambiente e as características pessoais que interferem na qualidade de vida. Assim, a CIF oferece um padrão de classificação que leva em consideração a presença e a gravidade de um problema de saúde, seja na vertente individual ou social? ${ }^{7}$.

A CIF utiliza um sistema de códigos alfanuméricos, onde a letra do nome do código refere-se a um determinado componente: "b" funções do corpo (body functions), "s" estruturas do corpo (structure), "d" atividade e participação (domain), e e" fatores ambientais (environmental factors). A parte numérica refere-se aos capítulos (ou categoria de primeiro nível) e categorias de segundo, terceiro e quarto nível, sendo os últimos os mais detalhados?

Para dar significado às categorias da CIF são atribuídos qualificadores genéricos, os quais indicam a gravidade do problema ou da barreira. Os qualificadores variam de 0 a 4 , no qual o 0 significa nenhum problema/facilitador, enquanto o 4 representa um problema/ facilitador completo. Há ainda os qualificadores 8 e 9, que significam "não especificado" e "não aplicável", respectivamente?

A aceitação e utilização da CIF como quadro de referência e classificação vem sendo facilitado pelo seu desenvolvimento e processo de consenso no mundo global, com crescente evidência sobre a sua validade ${ }^{8}$. Para aumentar a viabilidade do seu uso o projeto core sets começou a ser desenvolvido, cujo objetivo é o de estabelecer uma seleção de categorias adaptada para representar os padrões de avaliação multiprofissional de grupos específicos de pessoas ${ }^{9}$. O core set para indivíduos com TME crônico foi desenvolvido por 34 especialistas de 31 países diferentes. Na versão completa o core set é formado por 168 categorias de segundo, terceiro e quarto níveis. Já na versão abreviada estão inclusas 33 categorias de segundo nível ${ }^{10}$.

Como a CIF avalia os múltiplos aspectos da funcionalidade, acredita-se que essa ferramenta possa contribuir para avaliar adequadamente a funcionalidade de atletas com incapacidades. Assim, o objetivo deste estudo foi determinar o perfil funcional dos atletas com TME que treinam no time de BCR da cidade de Guarapuava-PR de acordo como o core set da CIF para indivíduos com TME.

\section{MÉTODOS}

Esta pesquisa caracterizou-se como um estudo transversal com atletas cadeirantes do time de basquete da cidade de Guarapuava, PR, Brasil. O estudo foi realizado entre agosto e setembro de 2012.

Foram incluídos no estudo os atletas do sexo masculino, com diagnóstico de lesão medular, com idade superior a 18 anos e que estivessem treinando há pelo menos três meses. Foram excluídos os atletas que não tinham diagnóstico clínico fechado da lesão. Assim, a amostra foi composta por 12 atletas, cujas características clínicas e socioeconômicas estão demonstradas na tabela 1.

Para determinação do perfil funcional dos atletas utilizou-se a versão abreviada do core set da CIF para indivíduos com TME crônico ${ }^{10}$.

A versão abreviada do core set da CIF para indivíduos com TME crônico contempla os quatro componentes da CIF, sendo composto por 33 categorias, das quais nove são de funções do corpo, quatro são de estruturas do corpo, 11 são de atividade e participação e nove são de fatores ambientais.

Para considerar a representatividade das categorias do core set, usou-se a recomendação de Jonsdottir et al. ${ }^{11}$, pela qual ao menos $20 \%$ dos pacientes com algum grau de limitação na categoria em questão faria com que esta fosse considerada válida. Isso significa que a somatória de indivíduos com qualificadores 1, 2, 3 ou 4 deveria ser igual ou maior que $20 \%$ da amostra para cada categoria ${ }^{12}$.

A classificação dos atletas foi realizada por meio de avaliações conduzidas por um profissional de saúde treinado e com experiência na utilização da CIF. Todos os atletas receberam informações sobre a participação no estudo e assinaram um termo de consentimento de acordo com a Resolução 196/96 do Conselho Nacional de Saúde. O estudo foi aprovado pelo Comitê de Ética da Universidade Estadual do Centro-Oeste, parecer 84090/2012.

Para análise dos dados foram calculadas as distribuições de frequências e as medianas dos qualificadores.

Tabela 1. Caracterização da amostra.

\begin{tabular}{|c|c|}
\hline Idade (anos), média (DP) & $35,5(7,2)$ \\
\hline \multicolumn{2}{|l|}{ Escolaridade, $\mathrm{n}(\%)$} \\
\hline Fundamental & $2(16,7 \%)$ \\
\hline Médio & $6(50 \%)$ \\
\hline Superior & $4(33,3 \%)$ \\
\hline \multicolumn{2}{|c|}{ Renda familiar (salários mínimos), n(\%) } \\
\hline 1 a 3 & $5(41,7 \%)$ \\
\hline 4 a 6 & $6(50 \%)$ \\
\hline 7 a 10 & $1(8,3 \%)$ \\
\hline \multicolumn{2}{|l|}{ Etiologia, $n(\%)$} \\
\hline Ferimento por arma de fogo & $4(33,3 \%)$ \\
\hline Queda de altura & $4(33,3 \%)$ \\
\hline Acidente de trânsito & $4(33,3 \%)$ \\
\hline \multicolumn{2}{|l|}{ Nível da lesão, n(\%) } \\
\hline Paraplégico & $12(100 \%)$ \\
\hline \multicolumn{2}{|l|}{ Tipo de lesão, n(\%) } \\
\hline Completa & $6(50 \%)$ \\
\hline Incompleta & $6(50 \%)$ \\
\hline \multicolumn{2}{|l|}{ Tempo de lesão, $n(\%)$} \\
\hline de zero até 5 anos & $3(25 \%)$ \\
\hline mais de 5 até 10 anos & $3(25 \%)$ \\
\hline mais de 10 até 15 anos & $4(33,3 \%)$ \\
\hline acima de 15 anos & $2(16,7 \%)$ \\
\hline
\end{tabular}

\section{RESULTADOS}

Os qualificadores 1, 2, 3 e 4, que representam níveis progressivos de deficiência, foram agrupados sob a coluna nomeada "alguma deficiência". Os qualificadores 0 e 9, que indicam, respectivamente, sem deficiência ou não aplicável, foram agrupados sob a coluna "sem deficiência". O qualificador 8 (não especificado) não foi utilizado, refletindo boa qualidade dos dados.

Assim, após a aplicação do core set foi possível demonstrar diversas deficiências e limitações relacionadas às funções e estrutura do corpo, atividades e participação, e fatores ambientais.

A tabela 2 apresenta a frequência das categorias das funções do 
corpo, na qual pode-se observar que apenas a categoria b710 (funções relacionadas à mobilidade das articulações) não foi considerada válida, pois apresentou mais de $80 \%$ da amostra sem comprometimento.

Entre as categorias dos componentes estruturas do corpo e atividade e participação, todas foram consideradas válidas (tabelas 3 e 4).

Já para o componente fatores ambientais, apenas a categoria e340 (cuidadores e assistentes pessoais) não foi considerada válida, pois 91,7\% da amostra não precisavam deste facilitador (tabela 5).

As categorias que todos os atletas apresentaram algum comprometimento foram: s120, d455, d465 e d530, enquanto para os fatores ambientais, todos consideram como facilitadores as seguintes categorias: e115, e150, e e580.

Já as categorias que apresentaram os maiores graus de acometimento, ou seja, que apresentaram mais de $50 \%$ dos atletas classificados pelos qualificadores 3 ou 4 foram: s120, d455 e d465. Para os fatores ambientais, mais da metade dos atletas precisava dos facilitadores e 115 e e 120 para auxiliar de maneira extrema ou total o desempenho de suas atividades.

Das 33 categorias do core set da CIF para indivíduos com TME crônico, 31 (93,9\%) puderam ser consideradas como representativas da amostra.

Tabela 2. Frequências das categorias das funções do corpo da CIF, de acordo com os qualificadores.

\begin{tabular}{c|c|c|c|c|c|c|c|c}
\hline \multirow{2}{*}{ Categoria da CIF } & \multicolumn{6}{|c}{ Qualificador da CIF } \\
\cline { 2 - 10 } & \multicolumn{1}{|c|}{ Alguma deficiência } & \multicolumn{3}{|c}{ Sem deficiência } \\
\hline & $\mathbf{1}$ & $\mathbf{3}$ & $\mathbf{4}$ & $\mathbf{0}$ & $\mathbf{0}$ & $\mathbf{9}$ & $\mathbf{\%}$ \\
\hline b152 Funções emocionais & 2 & 7 & - & - & 75,0 & 3 & - & 25,0 \\
\hline b280 Sensação de dor & 4 & 5 & 1 & 1 & 91,7 & 1 & - & 8,3 \\
\hline b525 Funções de defecação & 4 & 4 & - & 1 & 75,0 & 3 & - & 25,0 \\
\hline b620 Funções urinárias & 1 & 6 & - & 4 & 91,7 & 1 & - & 8,3 \\
\hline b640 Funções sexuais & 5 & 2 & 1 & 2 & 83,3 & 2 & - & 16,7 \\
\hline $\begin{array}{c}\text { b710 Funções relacionadas à } \\
\text { mobilidade das articulações }\end{array}$ & - & 1 & 1 & - & 16,7 & 10 & - & 83,3 \\
\hline $\begin{array}{c}\text { b730 Funções relacionadas } \\
\text { à força muscular }\end{array}$ & 4 & 2 & - & - & 50,0 & 6 & - & 50,0 \\
\hline $\begin{array}{c}\text { b735 Funções relacionadas } \\
\text { ao tônus muscular }\end{array}$ & 2 & 5 & 1 & 1 & 75,0 & 3 & - & 25,0 \\
\hline b810 Funções protetoras da pele & 1 & 3 & 1 & 1 & 50,0 & 6 & - & 50,0 \\
\hline
\end{tabular}

Tabela 3. Frequências das categorias das estruturas do corpo da CIF, de acordo com os qualificadores.

\begin{tabular}{|c|c|c|c|c|c|c|c|c|}
\hline \multirow{3}{*}{ Categoria da CIF } & \multicolumn{8}{|c|}{ Qualificador da CIF } \\
\hline & \multicolumn{5}{|c|}{ Alguma deficiência } & \multicolumn{3}{|c|}{ Sem deficiência } \\
\hline & 1 & 2 & 3 & 4 & $\%$ & 0 & 9 & $\%$ \\
\hline $\begin{array}{c}\text { s120 Medula espinal e estruturas } \\
\text { relacionadas }\end{array}$ & - & - & 6 & 6 & 100,0 & - & - & - \\
\hline s430 Estrutura do sistema respiratório & - & 4 & 1 & - & 41,7 & 7 & - & 58,3 \\
\hline s610 Estrutura do sistema urinário & 6 & 1 & - & 2 & 75,0 & 3 & - & 25,0 \\
\hline s810 Estruturas das áreas da pele & 2 & 1 & 1 & 1 & 41,7 & 7 & - & 58,3 \\
\hline
\end{tabular}

\section{DISCUSSÃO}

Atualmente tem-se demonstrado a importância de um estilo de vida fisicamente ativo para os indivíduos com $\mathrm{TME}^{13}$, pois o treinamento esportivo proporciona melhora na qualidade de vida destes indivíduos ${ }^{14,15}$. A atividade física adaptada também consegue prevenir complicações secundárias ${ }^{13}$ e pode complementar o processo de reabilitação de indivíduos que sofreram TME e necessitam de cadeira de rodas para mobilidade, além de representar novos objetivos e desafios na vida ${ }^{15}$.

Os primeiros sistemas de classificação de jogadores de BCR consideravam apenas a anatomia da lesão, porém, o atual sistema da IWBF inclui os aspectos funcionais dos atletas ${ }^{1}$. A proposta do uso da CIF não é a substituição deste sistema, mas associá-la para garantir uma classificação justa e equitativa entre os atletas e equipes.
Tabela 4. Frequências das categorias de atividade e participação da CIF, de acordo com os qualificadores.

\begin{tabular}{|c|c|c|c|c|c|c|c|c|}
\hline \multirow{3}{*}{ Categoria da CIF } & \multicolumn{8}{|c|}{ Qualificador da CIF } \\
\hline & \multicolumn{5}{|c|}{ Alguma deficiência } & \multicolumn{3}{|c|}{ Sem deficiência } \\
\hline & 1 & 2 & 3 & 4 & $\%$ & 0 & 9 & $\%$ \\
\hline d230 Realizar a rotina diária & 4 & 1 & - & - & 41,7 & 7 & - & 58,3 \\
\hline $\begin{array}{l}\text { d240 Lidar com o estresse e outras } \\
\text { demandas psicológicas }\end{array}$ & 3 & 5 & 2 & 1 & 91,7 & 1 & - & 8,3 \\
\hline $\begin{array}{c}\text { d410 Mudar a posição básica do } \\
\text { corpo }\end{array}$ & 5 & - & - & - & 41,7 & 7 & - & 58,3 \\
\hline d420 Transferir a própria posição & 3 & - & - & - & 25,0 & 9 & - & 75,0 \\
\hline d445 Uso da mão e do braço & 3 & 1 & - & - & 33,3 & 8 & - & 66,7 \\
\hline d455 Deslocar-se & 1 & 5 & 1 & 5 & 100,0 & - & - & - \\
\hline $\begin{array}{l}\text { d465 Deslocar-se utilizando algum } \\
\text { tipo de equipamento }\end{array}$ & 2 & 1 & 9 & - & 100,0 & - & - & - \\
\hline d470 Utilização de transporte & - & 6 & 2 & 2 & 83,3 & 2 & - & 16,7 \\
\hline d520 Cuidado das partes do corpo & 1 & 2 & - & - & 25,0 & 9 & - & 75,0 \\
\hline $\begin{array}{l}\text { d530 Cuidados relacionados aos } \\
\text { processos de excreção }\end{array}$ & - & 7 & 2 & 3 & 100,0 & - & - & - \\
\hline d550 Comer & 2 & 1 & - & - & 25,0 & 9 & - & 75,0 \\
\hline
\end{tabular}

Tabela 5. Frequências das categorias fatores ambientais da CIF, de acordo com os qualificadores.

\begin{tabular}{|c|c|c|c|c|c|c|c|c|}
\hline \multirow{3}{*}{ Categoria da CIF } & \multicolumn{8}{|c|}{ Qualificador da CIF } \\
\hline & \multicolumn{5}{|c|}{ Facilitador } & \multicolumn{3}{|c|}{$\begin{array}{c}\text { Nem facilitador } \\
\text { nem barreira }\end{array}$} \\
\hline & 1 & 2 & 3 & 4 & $\%$ & 0 & 9 & $\%$ \\
\hline $\begin{array}{l}\text { e110 Produtos ou substâncias para } \\
\text { consumo pessoal }\end{array}$ & 3 & 5 & - & 1 & 75,0 & 3 & - & 25,0 \\
\hline $\begin{array}{c}\text { e115 Produtos e tecnologia para } \\
\text { uso pessoal na vida diária }\end{array}$ & 3 & 3 & 1 & 5 & 100,0 & - & - & - \\
\hline $\begin{array}{c}\text { e120 Produtos e tecnologia para } \\
\text { mobilidade e transporte pessoal em } \\
\text { ambientes internos e externos }\end{array}$ & - & - & 1 & 8 & 75,0 & 3 & - & 25,0 \\
\hline $\begin{array}{l}\text { e150 Produtos e tecnologia usados } \\
\text { em projeto, arquitetura e construção } \\
\text { de edifícios para uso público }\end{array}$ & 1 & 6 & 3 & 2 & 100,0 & - & - & - \\
\hline $\begin{array}{l}\text { e155 Produtos e tecnologia usados } \\
\text { em projeto, arquitetura e construção } \\
\text { de edifícios de uso privado }\end{array}$ & 4 & 3 & 1 & - & 66,7 & 4 & - & 33,3 \\
\hline e310 Família imediata & - & 3 & 1 & 1 & 41,7 & 7 & - & 58,3 \\
\hline $\begin{array}{l}\text { e340 Cuidadores e } \\
\text { assistentes pessoais }\end{array}$ & - & - & - & 1 & 8,3 & 11 & - & 91,7 \\
\hline e355 Profissionais da saúde & 5 & 3 & - & - & 66,7 & 4 & - & 33,3 \\
\hline $\begin{array}{l}\text { e580 Serviços, sistemas e } \\
\text { políticas de saúde }\end{array}$ & 5 & 4 & 2 & 1 & 100,0 & - & - & - \\
\hline
\end{tabular}

Apesar do uso da CIF ainda ser incipiente, existe um crescente interesse na sua utilização ${ }^{16,17}$. Tal uso está em pleno processo de consolidação ao abordar a funcionalidade humana na sua totalidade, focando as possíveis relações e interações entre seus fatores condicionantes e determinantes, o que pode resultar em incapacidade ${ }^{18}$. Além disso, o potencial de crescimento da CIF é compatível com a demanda de conhecimento gerada pela mesma, tanto no setor público como no privado, ao envolver as relações entre saúde, funcionalidade e ambiente, principalmente no que se refere aos indicadores de saúde ${ }^{17}$, fatores totalmente relacionados à prática esportiva, como no BCR.

Nos casos de TME, a CIF é uma importante ferramenta, pois ela considera, a partir das funções do corpo, as formas e possibilidades de realização das atividades de vida diária e os fatores ambientais envolvidos, o que não ocorre com nenhum outro instrumento, pois não há ferramenta que contemple todas estas características ${ }^{3}$.

Foi utilizada a versão abreviada do core set por ser uma proposta inicial para uso da CIF com atletas, dando início, assim, às pesquisas sobre os aspectos multidimensionais da funcionalidade de atletas de BCR. 
Para validação das categorias optou-se pela frequência de 20\% de indivíduos com alguma deficiência, percentual semelhante ao utilizado para validação de categorias do core set para dor lombar ${ }^{11,12}$. Outro estudo sobre a aplicabilidade do core set para dor crônica em mulheres com fibromialgia ${ }^{19}$ utilizou a frequência de 30\% de não comprometimento da categoria, porém os próprios autores consideraram essa linha de corte pouco adequada.

Para a amostra analisada de atletas praticantes de BCR da cidade de Guarapuava-PR, a versão abreviada do core set da CIF para indivíduos com TME crônico permitiu a avaliação da funcionalidade de uma forma abrangente, incluindo as funções e estruturas do corpo, atividades e participação e fatores ambientais, pois 93,9\% das categorias foram consideradas representativas da amostra.

Apenas duas categorias não foram consideradas válidas para a amostra: $b 710$ (funções relacionadas à mobilidade das articulações) e e340 (cuidadores e assistentes pessoais).

A descrição categoria b710 é a seguinte: funções relacionadas à mobilidade de uma ou várias articulações, vertebral, ombro, cotovelo, cintura, quadril, joelho, tornozelo, pequenas articulações das mãos e pés; mobilidade generalizada das articulações; deficiências como hipermobilidade das articulações, rigidez articular, ombro "congelado", artrite $^{7}$. Para esse estudo, como a amostra foi composta por atletas com nível de lesão correspondente à paraplegia, foram consideradas as mobilidades da cintura escapular e dos membros superiores. Num primeiro momento acreditava-se que esse critério poderia ter interferido na não-inclusão desta categoria, pois os atletas demonstraram boa funcionalidade destes segmentos. Porém, para as categorias b730 (funções relacionadas à força muscular) e b735 (funções relacionadas ao tônus muscular) também foram considerados a cintura escapular e membros superiores, e estas categorias foram consideradas válidas. Acredita-se que tal fato demonstra a multidimensionalidade e multidirecionalidade da CIF, diferentemente do modelo linear de causa e efeito para as doenças e incapacidades.

Já a descrição da categoria e340 é a seguinte: indivíduos que fornecem os serviços necessários para dar suporte a outros indivíduos nas suas atividades diárias e na manutenção do desempenho no trabalho, educação ou outra situação da vida, fornecidos por meio de recursos públicos ou privados, ou em base voluntária, como provedores de suporte para construção e manutenção de casas, assistentes pessoais, assistentes de transporte, ajuda paga, babá ou outros que prestam cuidado ${ }^{7}$. Acredita-se que o nível da lesão e a prática regular de atividade física possam ter favorecido a maior funcionalidade destes atletas, deixando-os mais independentes de cuidadores, ou ainda que a melhor funcionalidade favoreceu a prática

\section{REFERÊNCIAS}

1. Gil-Agudo A, Arna-Espinoso, AD, Crespo-Tuitz B. Wheelchair basketball quantification. Phys Med Rehabil 2010;21(1):141-56.

2. Goosey-Tolfrey $\mathrm{V}$, Foden $\mathrm{E}$, Perret $\mathrm{C}$, Degens $\mathrm{H}$. Effects of inspiratory muscle training on respiratory function and repetitive sprint performance in wheelchair basketball players. Br J Sports Med 2012;44(9):665-8.

3. Vall J, Costa CMC, Pereira LF, Freisen TT. Application of International Classification of Functioning, Disability in Health (ICF) in individuals with spinal cord injury. Arq Neuropsiquiatr 2011;69(3):513-8.

4. Biering-Sørensen F, Hansen RB, Biering-Sørensen J. Mobility aids and transport possibilities 10-45 years after spinal cord injury. Spinal Cord 2004;42(12):699-706.

5. Whiteneck G, Meade MA, Dijkers M, Tate DG, Bushnik T, Forchheimer MB. Environmental factors and their role in participation and life satisfaction after spinal cord injury. Arch Phys Med Rehabil 2004;85(11):1793-803

6. Stucki G, Bickenbach JE, Post MWM. Developing epidemiologic studies of people's lived experience: the Swiss Spinal Cord Injury Cohort Study as a case in point. Am J Phys Med Rehab 2011;90(Suppl 2):S1-4.

7. Organização Mundial de Saúde (OMS). Organização Panamericana de Saúde (OPAS). CIF classificação internacional de funcionalidade, incapacidade e saúde. Universidade de São Paulo. São Paulo: Edusp, 2003.

8. Cieza A, Stucki G. The international classification of functioning disability and health: its development process and content validity. Eur J Phys Rehabil Med 2008;44(3):303-13.

9. Røe C, Sveen U, Geyh S, Cieza A, Bautz-Holter E. Construct dimensionality and properties of the categories in the ICF Core Set for low back pain. J Rehabil Med 2009;41(6):429-37.

10. Cieza A, Kirchberger I, Biering-Sørensen F, Baumberger M, Charlifue S, Post MW, Campbell R, Kovindha A, Ring H, Sinnott A, Kostanjsek N, Stucki G. ICF Core Sets for individuals with spinal cord injury in the long-term context. Spinal Cord 2010;48(4):305-12. desportiva. Também pode-se considerar as características socioeconômicas como um fator limitante para a contratação de cuidadores.

As categorias que todos os atletas apresentaram algum comprometimento foram: $\mathbf{1 2 0}$ (medula espinal e estruturas relacionadas), d455 (deslocar-se), d465 (deslocar-se utilizando algum tipo de equipamento) e d530 (cuidados relacionados aos processos de excreção). Esse comprometimento também pode ser observado nos seguintes fatores ambientes: e115 (produtos e tecnologia para uso pessoal na vida diária), e150 (produtos e tecnologia usados em projeto, arquitetura e construção de edifícios para uso público), e e580 (serviços, sistemas e políticas de saúde), pois todos os atletas relataram que precisavam destes facilitadores. Assim, pode-se observar que a CIF considera não apenas as disfunções e deficiências da pessoa, mas também o impacto destes fatores nas atividades sociais, assim como a influência do meio ambiente, seja como facilitador ou como barreira para a independência funcional.

Fato semelhante pode ser observado nas categorias com os maiores graus de comprometimento, pois das cinco categorias que apresentaram mais de $50 \%$ dos atletas classificados pelos qualificadores 3 ou 4, duas foram de atividade e participação: d455 (deslocar-se), d465 (deslocar-se utilizando algum tipo de equipamento); duas dos fatores ambientais: e 115 (produtos e tecnologia para uso pessoal na vida diária) e e120 (produtos e tecnologia para mobilidade e transporte pessoal em ambientes internos e externos); e uma do componente estruturas do corpo: s120 (medula espinal e estruturas relacionadas). Destaca-se que três destas cinco categorias estão relacionadas à locomoção.

Nas demais categorias os atletas apresentaram diferentes graus de incapacidade e limitação.

Este estudo limitou-se a uma amostra de atletas com diagnóstico de TME pertencentes a um time de BCR da cidade de Guarapuava-PR, o que não permite a generalização dos resultados, mas dá claros sinais de que a utilização da CIF pode e deve ser implementada em pesquisas semelhantes.

\section{CONCLUSÃO}

Assim, com o uso da versão abreviada da CIF para indivíduos com TME crônico, foi possível demonstrar que os atletas com TME do time de BCR da cidade de Guarapuava-PR, apresentaram diversos comprometimentos relacionados às funções e estruturas do corpo, atividades e participação e fatores ambientais, mas que não os impedem de realizar a prática esportiva.

Todos os autores declararam não haver qualquer potencial conflito de interesses referente a este artigo.
11. Jonsdottir J, Rainero G, Racca V, Glässel A, Cieza A. Functioning and disability in persons with low back pain. Disabil Rehabil 2010;32(Suppl 1):S78-84.

12. Riberto M, Chiappetta LM, Lopes KAT, Battistella LR. A experiência brasileira com o Core Set da Classificação Internacional de Funcionalidade, Incapacidade e Saúde para lombalgia. Coluna/Columna 2011;10(2):121-6.

13. de Groot S, van der Woude LHV, Niezen A, Smit CAJ, Post MWM. Evaluation of the physical activity scale for individuals with physical disabilities in people with spinal cord injury. Spinal Cord 2010;48(4):542-7.

14. Noce F, Simim MAM, Mello MT. A percepção de qualidade de vida de pessoas portadoras de deficiência física pode ser influenciada pela prática de atividade física? Rev Bras Med Esporte 2009;15:174-8.

15. Medola FO, Busto RM, Marçal AF, Achou Junior A, Dourado AC. O esporte na qualidade de vida de indivíduos com lesão da medula espinhal: série de casos. Rev Bras Med Esporte 2011;17(4):254-6.

16. Comín Comín M, Ruiz Garrós C, Franco E, Damian J, Ruiz Tovar M, de Pedro-Cuesta J. Scientificprofessional production on the ICF disability model in Spain. A literature review (2001-2010). Gac Sanit 2011;25(2):39-46

17. Ruaro JA, Ruaro MB, Souza DE, Fréz AR, Guerra RO. Panorama e perfil da utilização da CIF no Brasil uma década de história. Rev Bras Fisioter. 2012;16(6):454-62.

18. Sampaio RF, Luz MT. Funcionalidade e in capacidade humana: explorando o escopo da classificação internacional da Organização Mundial da Saúde. Cad Saúde Pública 2009;25(3):475-83.

19. Riberto M, Saron TRP, Battistella LR. Resultados do core set da CIF para dor crônica generalizada em mulheres com fibromialgia no Brasil. Acta Fisiatr 2008;15(1):6-12. 